\title{
Explanation of Expansion of Universe On The Basis Of Theory of Relativity and Consideration of Multiverse and Anti Gravity
}

\author{
Rishabh Mishra \\ School of Naval Architecture and Ocean Engineering Indian Maritime University
}

Abstract: This paper is all about producing a new way to analyse the expansion of universe with some different perspective rather than continuing with the mystry of dark energy.

\section{A Simple But Grand Example}

Before explanation of why the universe is expanding we need consider a simple but most important example that is following. Let Us Consider Wheel Which Is Assumed To Be A Perfect Circle And It Is Observed By Two Observers One Is In A Frame Which Is At Infinite Distance From The Rim And Another Observer In Such Aframe That He Is Able To See Every Action Of The Rim Without Length Contraction And Without Time Contraction And He Is Able To See The The Original Dimensions Of The Rim. So The Name Of First Frame I Mentioned Could Be "F1" And Second Frame Is "F2"

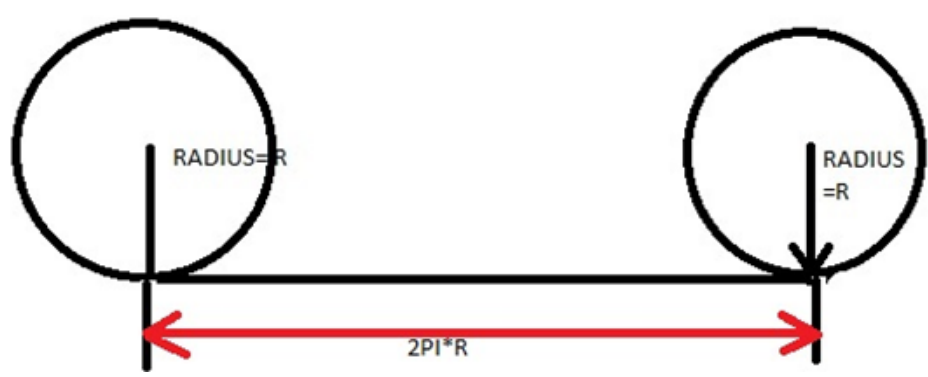

Let the Area of Rim Is =A

Let the Radius of Wheel Is $=\mathrm{R}$

Case 1:

When the Observer Is In Frame Of Reference "F2" That Is Very Near To the Rim

If The Rim Rotated And Just Moved So That It Completed Its One Rotation He Observed That Its Centre Of Mass Moved By A Distance Of " $2 \pi r$ ”.

And The Area Of Disc That He Observed Is "A".

Case 2:

When The Person At Infinity Is Oberving This Rim From Infinity.(From Frame "F1")

Mathematicaly We Know That "A Straight Line Is an Arc of Circle Whose Radius Is At Infinity"

This Concept Will Be Followed Here and Let Us See How This Motion of the Rim Will Be Seen From the Infinity (Or Frame F2)

Let The Path Of The Centre Of The Rim Sutends Some Angle "A" At The Centre Of This Infinite Circle.

By Using Geometry We Can Find the Angle It Subtends At the Centre As Following

$\mathrm{A}=(2 \pi \mathrm{r}) / \infty$

So, $A=0$

Also The Rim Will Look Like A Point From The Frame Of Reference "F1".

Mathematicaly We Can Explain It Following

Let the Solid Angle Formed By the Area of the Ring Is " $\Omega$ "

We Know That

$\Omega=\mathrm{A} / \mathrm{R}^{2}$

Here for Observer at Frame F1

$\mathrm{R}=\infty$

Hence

$\Omega=\mathrm{A} / \infty^{2}$

$\Omega=0$.

We Can Say That Solid Angle of A Point Is Only =0

DOI: 10.9790/4861-0903020407

www.iosrjournals.org 
"So the Object Will Be Oserved likes A Point"

Because For Corresponding Linear Motion Of The Rim The Angular Displacement Observed At The Infinity Is "0 Rad" And If The Observed Angular Displacement Is Zero Then Te Observed Linear Motion Must Also Be Zero.

Hence We Can Conclude That The Person At Infinity Does Not Observed Any Motion And For Him The Rim Was Stationary For Him.

Case: 3

Now Consider a Frame Of Reference In Which If An Observer Is Oscillating Between These Two Frame Of References

- In The Condition If This Frame Is Moving Towards The Rim The Observer Will See The Rim Is Accelerating In Its Motion.

- In The Condition If This Frame Is Moving Away From Rim It Will Observe The Rim Deccelerated Motion.

And This Same Phenomena Is Applying On Our Universe

We Are In Such A Frame Of Reference That Is Also Oscillating Between Such Two Frame Of References.

I saying this not only on theoretical background but also I have practical evidence

"Scientists Have Found That Our Universe Have Started Accelerating 5billion Years Ago But From Which Frame, From The Frame Of Earth And Also They Have Cocluded That Before That Universe Was Slowing Down And That Observation Is Also Done From The Frame Of Reference Of Earth From This Only I Can Conclude That The Motion Of Our Frame Must Have Effects On Our Observations.”

\section{Detailed Explanation On The Basis Of Theory Of Relativity}

As per the Einstien's eqations it is confirmed that the universe is either expanding or contracting .It cannot be treated as a stationary

(But the Question Comes Into Picture Is 'With Whose Respect?' In This Relativistic World).

It has been an observed phenomena that the the space is expanding but space and time are not two independent quantities in this 'relativistic world' so the time must also expand in better word I should say the flow of time for rest of universe frame should also increase (here from rest of universe frame I mean frame of universe except earth frame).

What all observation that our physicist have done till now all the observation are done from earths frame of reference that means what all results we got are with respect to earths frame of reference.

So the possibility is there that from different frame of reference if we observe the universe we will get different results. It is possilble that if for one frame of reference universe looks to be expanding it is possible that for another frame of reference it may look still and for another it may look contracting just in order to keep the velocity of light(c) constant as per the theory of relativity.

\section{Explanation}

This is well known fact that this universe is basically a space time fabric and all these phenomena are taking place on this fabric. If look at the Einstiens special theory of relativity. I propose that we are moving at very high velocity (comparable to the the velocity of light) a way (i.e. opposite to the direction of light in all three dimensions) that we are observing the expansion of universe hence keeping this state of motion if our frame moves we will observe the expansion of universe. But it has been observed that our universe is expanding with an accelerated motion so this means that we are also accelerating along our path (i.e having either circular or elliptical motion).

NOW I am going to make daring statement

"There no dark energy in this universe"

I dared to make such a statement because concept of relativity can itself explains this phenomena there is no such need of considering a hypothetical energy i.e. dark energy.

\section{Final Conclusion}

“Universe Is Not Expanding Because Of Some Mysterious 'Dark Energy'. It Is Expanding 'Relative' To Our Frame Of Reference Because Our State Of Motion Is Such That In Order To Keep The Velocity Of Light Constant The Space And Time Both Are Expanding Themselve With Acceleration (Because We Are Also Accelerating Relative To 'Rest Of Universe Frame') .

The Acceleration Here Is Due To Oscillatory Motion Of Our Frame Of Reference. 


\section{Reasons For Discarding The Existance Of Dark Energy}

Let us assume that dark energy is causing the expansion of this universe. As dark energy is also an energy so it will also follow the law of conservation of energy.

If space is expanding it must have some kinetic energy and if dark energy is the only cause for this expansion then this dark energy is converting its form to kinetic energy.

A well known and peculiar feature of expansion of universe is that it is expanding with acceleration but it is known that a body can only accelerate if an only if extra energy is provided to it. This means that dark energy cannot remain constant it should increase with time. But the total energy of the universe is constant and even if this dark energy is increasing that means all other forms of energy like all celestial bodies, dark matter and all other forms of energy in this universe should be decreasing in their amount with time but I do not think there is something happening like this in this universe.

\section{Mathematical Explanation}

Let I choose a point mass of rest mass ' $\mathrm{m}$ ' which is a part of expanding universe is moving with some velocity ' $u$ ' which is very small with respect to the velocity of light.

Hence we can write the total energy of the mass as follows:

$\mathrm{E}=\mathrm{mc}^{\wedge} 2+(1 / 2) \mathrm{mu}^{\wedge} 2 \quad \ldots(1)$

But after getting accelerated to some velocity ' $\mathrm{v}$ ' it will have kinetic energy as follows:

$\mathrm{E}^{\prime}=\mathrm{mc}^{\wedge} 2+(1 / 2) \mathrm{mv}^{\wedge} 2 \quad \ldots(2)$

If we subtract equation (1) from (2) we can easily get the energy required by this mass to getting it accelerated from velocity 'u' to ' $v$ '.

Hence we get the following result

$\Delta \mathrm{E}=(1 / 2) \mathrm{m}\left(\mathrm{v}^{\wedge} 2-\mathrm{u}^{\wedge} 2\right) \quad$...(3)

The above mentioned change in energy of given mass is responsible for for its acceleration.

So for whole universe the extra energy needed for getting this acceleration can be given by

TOTAL ENERGY $=\sum(\Delta \mathrm{E}) \quad \ldots(4)$

While doing all the above calculation I have not taken in account the gravitational interaction between the bodies.

The real picture of expansion of universe is given by the kinetic energy and also with passage of time the gravitational interaction between the bodies will also decrease as because of expansion of universe the gap between them will increase and hence the gravitational pull between them will decease.

\section{Contradiction}

If other forms of energy in this universe are not changing to dark energy. Then how this dark energy is increasing. For this we can consider that may be universe is creating energy by some mechanism but this is directly contradictory to the fact that "ENERGY CANNOT BE CREATED NOR BE DESTROYED". This means that may be universe is taking energy from some external source and seems to be hypothetical (Possibility Is There That It May Be True If The Case Of Multiverse Is Taken Into Consideration). "So I Propose That The Cosideration Of Dark Energy Is Just Hypothetical Until We Consider The Phenomena Of Multiverse. And If The Concept Of Multiverse Is Not True Then In That Case The Only Possible Concept Of Relativity Can Explain The Phenomena Of Expansion Of Universe In Some What The Way I Have Mentioned Above".

\section{Reason for Discarding the Phenomena of Anti Gravity as a Cause for Expansion of Universe}

Anti-gravity is just a hypothetical force (no such phenomena have been found yet). But for the shake of understanding we can define anti-gravity in following manner.

"Anti-gravity is just a phenomena opposite to the phenomena of 'GRAVITY'."

But it is impossible that it can act between the masses because if it acted then in that case it will cause repulsion between the masses. But according to Einstiens "GENERAL THEORY OF RELATIVITY" a body with some mass always make some indentation in the space time fabric an if this is true it is impossible that two bodies with certain masses will repel each other(if total charge on both the bodies is zero and gravitation is only acting between them). So upto my thinking level I can discard the phenomena of anti gravity just by using the "THE GENERAL THEORY OF RELATIVITY”.

\section{Possibilities Of Anti Gravity}

In our 'world' there could be no such phenomena of anti-gravity but if we consider a case of a 'parallel world where every phenomena of this 'world' is taking place in just an opposite manner.

And if it is so then the theory of relativity will be valid there also but will work in just the opposite sense it works in our 'world'. 
Hence in that case the phenomena of anti-gravity are valid.

But as per the present understanding of physics we cannot go in such parallel world because for that we need to cross the speed of light as per the

\section{Light Cone}

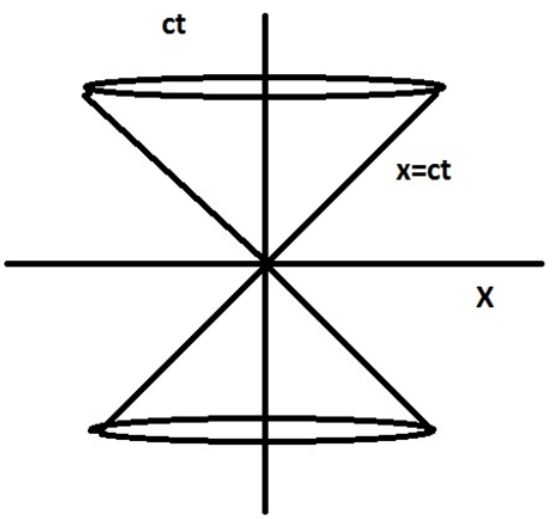

'RELATIVITY EQATIONS'. Which seems to be impossible (I used 'seems to 'because I donot know the future).

"We can see the 'space time cone' and we can conclude that only a particular space we can travel so we can say that the rest space except this infinite cone is the world that is anti-parallel to us."

"So I Finaly Propose That Universe Is Expanding With Some Constant Rate As It Ever Was. It Is Only The Motion Of Our Frame Which Has Made Us To Observe That Between Certain Period Of Time The Expansion Of Universe Is Slowing Down And Between Certain Period Of Time It Is Accelerating Up (Which Is Continuing Till Now Also)" 